\title{
Sympathetic cooling of molecular ions in a surface-electrode ion trap
}

\author{
A. Mokhberi and S. Willitsch* \\ Department of Chemistry, University of Basel, Klingelbergstrasse 80, 4056 Basel, Switzerland
}

(Received 16 April 2014; published 4 August 2014)

\begin{abstract}
We report the sympathetic cooling and Coulomb crystallization of molecular ions above the surface of an ion-trap chip. $\mathrm{N}_{2}^{+}$and $\mathrm{CaH}^{+}$ions were confined in a surface-electrode radio-frequency (rf) ion trap and cooled by the interaction with laser-cooled $\mathrm{Ca}^{+}$ions to secular translational temperatures in the millikelvin range. The configuration of trapping potentials generated by the surface electrodes enabled the formation of planar bicomponent Coulomb crystals and the spatial separation of the molecular from the atomic ions on the chip. The structural and thermal properties of the Coulomb crystals were characterized using molecular dynamics simulations. The present study extends chip-based trapping techniques to cold molecular ions with potential applications in mass spectrometry, chemistry, quantum information science, and spectroscopy.
\end{abstract}

DOI: 10.1103/PhysRevA.90.023402

PACS number(s): 37.10.Pq, 37.10.Mn, 37.10.Ty

The recent development of miniaturized trapping devices providing tightly confining, highly flexible trapping potentials has paved the way for new schemes to control neutral atoms and atomic ions. The ability to trap and cool neutral atoms on the surface of microstructured chips has enabled new experiments in, e.g., quantum optics, quantum interferometry, and metrology [1-3]. Similarly, the development of rf ion-trap chips [4-6] has laid the basis for improved protocols for the manipulation of ions, which is of importance for, e.g., quantum information processing [7,8] and quantum simulation [9-11].

While chip techniques for atomic species are by now well established, their extension to molecules has proven challenging. Translationally cold molecules and molecular ions are currently of great interest for applications in precision spectroscopy [12,13], cold chemistry and collision studies [14-17], quantum optics [18], and quantum information science $[19,20]$. However, significant difficulties arise because their complex molecular energy-level structure precludes the implementation of closed optical transitions in most cases. Therefore, standard techniques of atomic physics like laser cooling and fluorescence detection of single particles are at best only applicable to a very restricted number of molecular systems [21]. For polar neutral molecules such as CO and Rydberg atoms, the deceleration and trapping on a chip has only recently been achieved using time-varying inhomogeneous electric fields [22-24].

Here, we report the generation of Coulomb crystals, i.e., ordered structures of translationally cold molecular ions, above the surface of an ion-trap chip. The molecular ions were cooled sympathetically by their interaction with simultaneously trapped laser-cooled atomic ions [25,26]. The trapping potential configuration generated by our chip enabled the formation of two- and three-dimensional bicomponent Coulomb crystals as well as the spatial separation of both species in layers parallel to the chip surface.

The present work paves the way for improved protocols for the control of the external and internal degrees of freedom of molecular ions using accurately shaped external fields in miniaturized trapping devices, extending previous developments for atomic species [8] to molecules. It also opens up

*stefan.willitsch@unibas.ch perspectives for the development of highly integrated experiments with molecular ions combining various tasks such as ion preparation, mass spectrometry, chemistry, and spectroscopy which have thus far been the domain of costly and complex guided-ion beam machines [27] in a monolithic miniaturized device. The compact, flexible, and easily accessible trapping structures for cold molecular ions demonstrated here will also facilitate their integration with other quantum systems such as neutral atoms [16], neutral molecules [14], or nanomechanical oscillators. The technology demonstrated here thus has the potential to benefit a wide range of fields including quantum technology, chemistry, mass spectrometry, and spectroscopy.

Inspired by Ref. [28], we adopted a six-wire surfaceelectrode (SE) ion trap consisting of a split central electrode, two rf electrodes, and two batteries of control and endcap electrodes; see Fig. 1. The rf electrodes were used to generate time-varying electric fields for the dynamic trapping of the ions in the transverse $(x, y)$ directions [26]. All other electrodes were used to apply static (dc) voltages to confine the ions in the longitudinal $(z)$ direction, to tilt the principal axes of the trap for efficient laser cooling of the atomic ions [28], to compensate for excess micromotion [29], and to shape the trapping potentials in order to manipulate the structure of the Coulomb crystals [10]. The width of the rf and central electrodes amounted to $2.00 \mathrm{~mm}$ and $750 \mu \mathrm{m}$, respectively. The width of the gaps between the electrodes was $300 \mu \mathrm{m}$. The trap electrodes were laser cut out of a $0.50-\mathrm{mm}$ stainless-steel foil, electropolished, and coated with a layer of gold with a nominal thickness of $2 \mu \mathrm{m}$. The trap was glued on a ceramic frame separating the electrodes from a printed circuit board containing the capacitors for the $R C$ low-pass filters isolating the dc electrodes from the rf. This specific trap layout was chosen in order to avoid any dielectric surfaces in the vicinity of the trap center which could cause the buildup of stray charges and patch potentials.

To load the trap with atomic ions, a beam of calcium atoms was produced by evaporation from a resistively heated stainless-steel tube underneath the central trap region. The beam passed through a $300-\mu \mathrm{m}$-wide slit skimmer, the ceramic base of the trap, and finally the gap between the two central electrodes to reach the trap center where $\mathrm{Ca}^{+}$ions were produced by nonresonant photoionization; see Fig. 1. This 


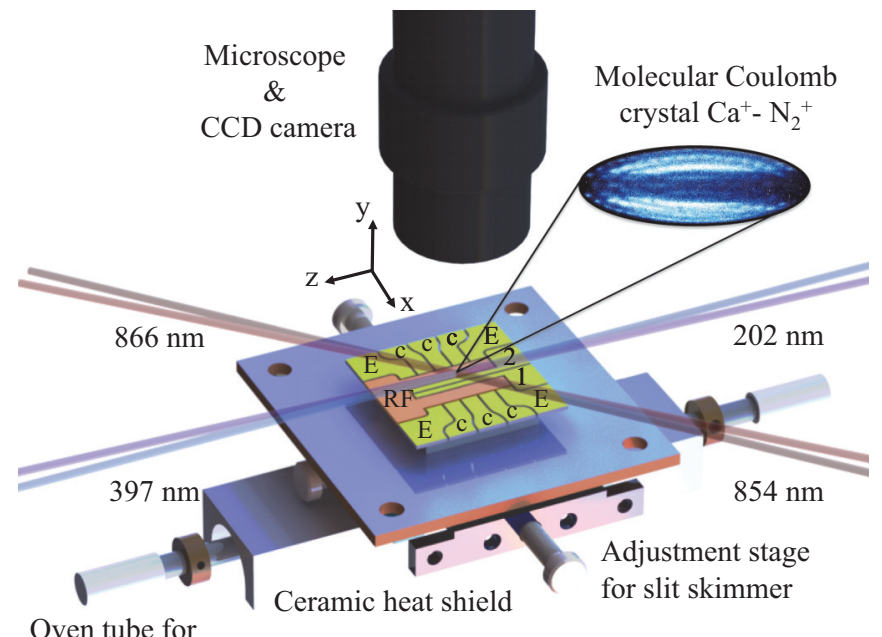

backside loading of $\mathrm{Ca}$

FIG. 1. (Color online) Experimental setup and layout of the surface-electrode ion trap. The electrodes are labeled with $R F$ for the rf electrodes, 1 and 2 for the two central dc electrodes, $E$ for dc endcap electrodes, and $c$ for dc control electrodes. The laser beams $(397,866$, and $854 \mathrm{~nm}$ for laser cooling $\mathrm{Ca}^{+}$and $202 \mathrm{~nm}$ for photoionizing $\mathrm{N}_{2}$ and $\mathrm{Ca}$ ) were introduced parallel to the chip surface and superimposed in the center of the trap. The inset shows a fluorescence image of a $\mathrm{Ca}^{+}-\mathrm{N}_{2}^{+}$bicomponent Coulomb crystal.

backside loading technique has the advantage of avoiding the coating of the chip surface with $\mathrm{Ca}$ deposited from the beam [30]. Doppler laser cooling of $\mathrm{Ca}^{+}$ions was achieved using three diode laser beams at 397, 866, and $854 \mathrm{~nm}$ pumping on the $(4 s)^{2} S_{1 / 2} \rightarrow(4 p)^{2} P_{1 / 2},(3 d)^{2} D_{3 / 2} \rightarrow(4 p)^{2} P_{1 / 2}$, and $(3 d)^{2} D_{5 / 2} \rightarrow(4 p)^{2} P_{3 / 2}$ transitions.

For the sympathetic-cooling experiments, we chose $\mathrm{N}_{2}^{+}$ (mass $28 \mathrm{amu}$ ) and $\mathrm{CaH}^{+}$(mass $41 \mathrm{amu}$ ) as prototypical molecular ions which have a lighter and a heavier mass compared to the laser-cooled $\mathrm{Ca}^{+}$ions (mass $40 \mathrm{amu}$ ), respectively. The $\mathrm{N}_{2}^{+}$ions were produced above the surface of the chip by resonance-enhanced $(2+1)$-photoionization (REMPI) via the $a^{\prime \prime} \Sigma_{g}^{+}$intermediate electronic state of neutral $\mathrm{N}_{2}$ [31] introduced into the vacuum chamber through a leak valve at partial pressures $<6 \times 10^{-9}$ mbar. REMPI was carried out using the frequency-tripled output of a pulsed dye laser at a wavelength of $202 \mathrm{~nm}$ and a pulse energy of $80 \mu \mathrm{J}$. $\mathrm{CaH}^{+}$ions were produced by chemical reactions of laser-excited $\mathrm{Ca}^{+}$ions with $\mathrm{H}_{2}$ molecules leaked into the vacuum chamber. The fluorescence of the $\mathrm{Ca}^{+}$ions was spatially resolved by a microscope (magnification $\approx 11.5$ ) and imaged onto a charge-coupled device (CCD) camera with a resolution of $2 \mu \mathrm{m}$.

The operation of the trap can be understood in terms of an adiabatic approximation [27] in which the total effective trapping potential $\Phi_{\mathrm{t}}$ is given by the sum of a time-independent pseudopotential $\Phi_{\mathrm{ps}}$ and a static potential $\Phi_{\mathrm{dc}}$ generated by the trap electrodes:

$$
\begin{aligned}
\Phi_{\mathrm{t}}(x, y, z) & =\Phi_{\mathrm{ps}}+\Phi_{\mathrm{dc}} \\
& =\frac{Q^{2} V_{\mathrm{rf}}^{2}}{4 M \Omega_{\mathrm{rf}}^{2}}\left\|\nabla \phi_{\mathrm{rf}}\right\|^{2}+Q \sum_{i} V_{i} \phi_{i, \mathrm{dc}} .
\end{aligned}
$$

(a)

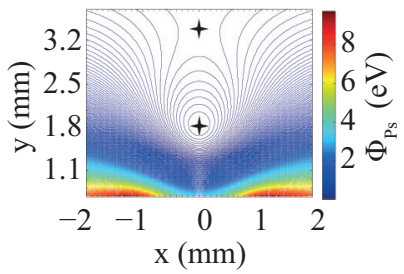

(c)

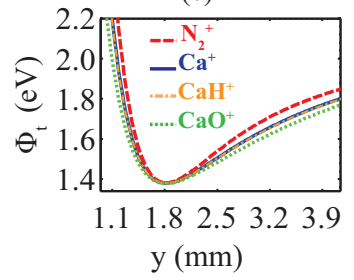

(b)

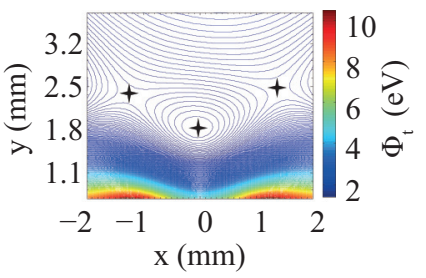

(d)

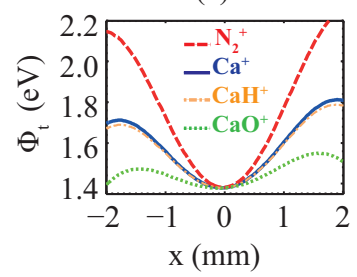

FIG. 2. (Color online) Calculated trapping potentials: (a) Pseudopotential $\Phi_{\mathrm{ps}}$ and (b) total potential $\Phi_{t}$ for $\mathrm{Ca}^{+}$ions in the $(x, y)$ plane intersecting the trap center perpendicular to the trap axis. The crosses indicate the positions of the potential minima and saddle points. (c, d) One-dimensional cuts through $\Phi_{t}$ at the trap center for $\mathrm{N}_{2}^{+}, \mathrm{Ca}^{+}, \mathrm{CaH}^{+}$, and $\mathrm{CaO}^{+}$along the $y$ and $x$ axes, respectively, illustrating the mass dependence of the trapping potential. The potential configuration shown results from the parametrization procedure of Ref. [28] described in the text. The small asymmetry of the potential results from a slight tilt of the principal axes for more efficient laser cooling of the ions in the $(x, y)$ plane if required. See Fig. 1 for the definition of the coordinate axes.

Here, $Q$ and $M$ are the charge and mass of the trapped ions, respectively, and $V_{\mathrm{rf}}$ and $\Omega_{\mathrm{rf}}$ denote the $\mathrm{rf}$ amplitude and frequency, respectively. The potential terms $\phi_{\mathrm{rf}}$ and $\phi_{i, \mathrm{dc}}$ are the solution of the Laplace equation for a unit voltage applied to the $\mathrm{rf}$ and the $i$ th dc electrode, respectively. $V_{i}$ is the voltage applied on the $i$ th dc electrode.

The trapping potentials were calculated by solving the Laplace equation numerically using finite element methods (FEMs) as well as using analytical representations of the electrode potentials [32,33]; see Fig. 2. The height of trapping, defined as the vertical position of the minimum of the total potential, was calculated to be $1.82 \mathrm{~mm}$ above the surface. The depth of the pseudopotential $\Phi_{\mathrm{ps}}$, defined as the potential difference between the minimum and the saddle point above the surface through which ions can escape [see Fig. 2(a)], was calculated to be $117 \mathrm{meV}$ for $\mathrm{Ca}^{+}$ions at the rf voltage $V_{\mathrm{rf}}=495 \mathrm{~V}$ and frequency $\Omega_{\mathrm{rf}}=2 \pi \times 8.0 \mathrm{MHz}$ used in the experiments.

To determine the optimal static voltages applied to the dc electrodes, the parametrization method of Ref. [28] was used. This procedure ensured that the minimum of the total potential for $\mathrm{Ca}^{+}$always coincided with the position of the rf null line (the axis on which the rf fields vanish) in order to avoid excess micromotion. Typical voltages used in the experiments were $\{$ central $(1,2)$, control (c), endcap (E) electrodes $\}=\{2$ to $2.3,-6$ to $-6.6,21.5$ to 23$\} \mathrm{V}$. The application of the static potentials $\Phi_{\mathrm{dc}}$ gave rise to saddle points of $\Phi_{\mathrm{t}}$ of center along the $x$ axis providing additional escape routes of the ions from the trap [see Fig. 2(b)]. For $\mathrm{Ca}^{+}$, the effective trap depth limited by the 
escape of the ions through these saddle points was calculated to be $113 \mathrm{meV}$.

Using the same trapping parameters, the total trapping potential $\Phi_{\mathrm{t}}$ has also been calculated for $\mathrm{N}_{2}^{+}, \mathrm{CaH}^{+}$, and $\mathrm{CaO}^{+}$ ions. From Eq. (1), it can be seen that the pseudopotential $\Phi_{\mathrm{ps}}$ is inversely proportional to the ion mass, leading to a segregation of the ion species in the Coulomb crystals. This situation is completely analogous to the one found in conventional linear rf traps [26]. Figures 2(c) and 2(d) show one-dimensional cuts of $\Phi_{\mathrm{t}}$ at the trap center along the $y$ and $x$ axes, respectively, for $\mathrm{N}_{2}^{+}, \mathrm{CaH}^{+}$, and $\mathrm{CaO}^{+}$ions in comparison with $\mathrm{Ca}^{+}$. For the $\mathrm{N}_{2}^{+}$and $\mathrm{CaH}^{+}$ions used in the experiments, the effective trap depths were calculated to be 261 and $104 \mathrm{meV}$, respectively.

To characterize the thermal and structural properties of the Coulomb crystals observed in the experiments, we performed molecular dynamics (MD) simulations [26,34,35]. Briefly, the classical equations of motion for the laser-cooled and sympathetically cooled ions in the trap were solved numerically. The total force acting on the ions was expressed by

$$
\mathbf{F}_{\text {tot }}=\mathbf{F}_{\text {trap }}+\mathbf{F}_{\text {Coulomb }}+\mathbf{F}_{\mathrm{LC}}+\mathbf{F}_{\text {heating }}+\mathbf{F}_{\mathrm{RP}} \text {, }
$$

where the terms on the right-hand side represent the trapping force, the Coulomb force between ions, the laser-cooling force, an effective heating force (reflecting collisions with background gas molecules and imperfections of the setup), and the radiation pressure force generated by unidirectional laser cooling $[34,35]$. The laser-cooling and radiation pressure forces act only on the atomic ions, while the trapping, Coulomb, and heating forces act on both species. A careful comparison of the simulated and experimental images served to determine the number of the ions in the crystal and their kinetic energies [35]. To calculate the trapping forces, threedimensional Taylor expansions were fitted to the numerical electrode potentials. The expansion was truncated after the fourth order and reproduced the numerical potentials within $0.001 \%$ in a cube of $400 \mu \mathrm{m}$ length around the trap center. The trap force $\mathbf{F}_{\text {trap }}$ was calculated from analytical gradients of the fitted potential functions. In the simulations, fully time-dependent potentials were used so that effects related to the ion micromotion could be characterized.

Based on extensive comparisons between the experimental images and sets of simulations in which the ion numbers and secular energies, i.e., the thermal ion energies without micromotion, have been varied, we estimate the uncertainty in the determination of the ion numbers to be \pm 1 for molecular ions and $\pm 2( \pm 5)$ for the $\mathrm{Ca}^{+}$ions in small (big) crystals.

Figure 3 shows experimental and simulated images of typical Coulomb crystals imaged in the $(x, z)$ plane parallel to the chip surface. The molecular ions do not fluoresce and are only indirectly visible as dark areas in the crystal images. In Fig. 3(c), the sympathetically cooled molecular ions have been made visible in the simulations for clarity. Column (I) in Fig. 3 shows a pure atomic Coulomb crystal containing $262 \mathrm{Ca}^{+}$ions at a mean secular energy $E_{\mathrm{sec}} / k_{B}=23 \mathrm{mK}$. Although the shapes of the crystals projected onto the imaging plane are reminiscent of those obtained in conventional linear rf traps [26], the crystals in the SE trap are not spheroidal owing to the lower symmetry of the trapping potentials [10]. This is also reflected by the lack of degeneracy of the trap frequencies in the transverse directions. For the present case, the principal

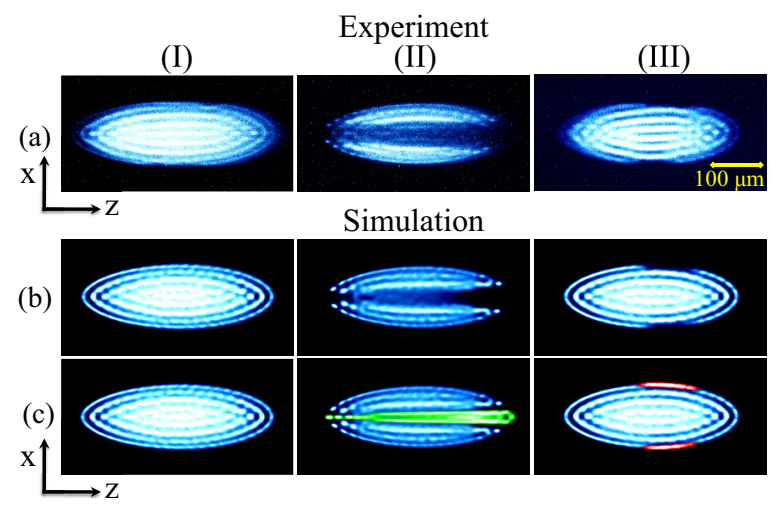

FIG. 3. (Color online) (a) Experimental and (b, c) simulated false-color laser-cooling fluorescence images of bicomponent Coulomb crystals on the chip. In (c), the molecular ions were made visible in the simulations for clarity. Color code: $\mathrm{Ca}^{+}$, blue; $\mathrm{N}_{2}^{+}$, green [in the center of the crystal]; and $\mathrm{CaH}^{+}$, red [at the extremities of the crystal]. (I) Pure atomic crystal containing $262 \mathrm{Ca}^{+}$ions, (II) 50 sympathetically cooled $\mathrm{N}_{2}^{+}$and $180 \mathrm{Ca}^{+}$ions, and (III) 7 sympathetically cooled $\mathrm{CaH}^{+}$and $200 \mathrm{Ca}^{+}$ions. See text for details.

trap frequencies have been calculated to be $\omega_{x} / 2 \pi=171$, $\omega_{y} / 2 \pi=272$, and $\omega_{z} / 2 \pi=83 \mathrm{kHz}$.

The $\mathrm{Ca}^{+}-\mathrm{N}_{2}^{+}$bicomponent Coulomb crystal shown in Fig. 3(a) [column (II)] was obtained by loading $\mathrm{N}_{2}^{+}$into a $\mathrm{Ca}^{+}$Coulomb crystal as described above. Based on the MD simulations, we inferred that this crystal contains 50 $\mathrm{N}_{2}^{+}$ions sympathetically cooled to $E_{\mathrm{sec}} / k_{B}=43 \mathrm{mK}$ in equilibrium with $180 \mathrm{Ca}^{+}$at $E_{\mathrm{sec}} / k_{B}=33 \mathrm{mK}$. Since the trapping potential is steeper for lighter ions [see Figs. 2(c) and 2(d)], the $\mathrm{N}_{2}^{+}$ions localized closer to the central trap axis than the $\mathrm{Ca}^{+}$ions. The asymmetric shape of the $\mathrm{N}_{2}^{+}$crystal along the $z$ direction was caused by the unidirectional radiation pressure force acting on the $\mathrm{Ca}^{+}$ions as well as small asymmetries in the potential induced by the geometry of the rf electrodes.

Column (III) of Fig. 3 shows $7 \mathrm{CaH}^{+}$ions at $E_{\mathrm{sec}} / k_{B}=$ $28 \mathrm{mK}$ sympathetically cooled by $200 \mathrm{Ca}^{+}$ions at $E_{\mathrm{sec}} / k_{B}=$ $24 \mathrm{mK}$. The heavy $\mathrm{CaH}^{+}$ions localize at the edges of the $\mathrm{Ca}^{+}$crystal. We found it difficult to cool a larger number of $\mathrm{CaH}^{+}$ions, which might be due to the reduced efficiency for the sympathetic cooling of heavy ions at the extremities of the crystal [36], the reduced trap depth for $\mathrm{CaH}^{+}$[see Fig. 2(d)] which facilitates the escape of the initially hot molecular ions produced by chemical reactions, micromotion heating, or a combination of these.

The flexibility in shaping the trapping potentials on the chip lends itself to a precise manipulation of the crystals to form structures which are challenging to obtain in conventional electrode geometries. In the crystal in Fig. 4, column (I), the molecular and atomic ions were pushed away from the rf null line by decreasing the voltages applied to the central electrodes. Because of the mass dependence of the pseudopotential, the two ion species were spatially separated along the $y$ axis such that the $\mathrm{N}_{2}^{+}$ions were located closer to the central trap axis and the $\mathrm{Ca}^{+}$ions farther away. The result were two layers of ions with a mean distance of $19 \pm 2 \mu \mathrm{m}$ according to the MD simulations as can be seen in the side view of the simulated crystal in Fig. 4(d), column (I). 


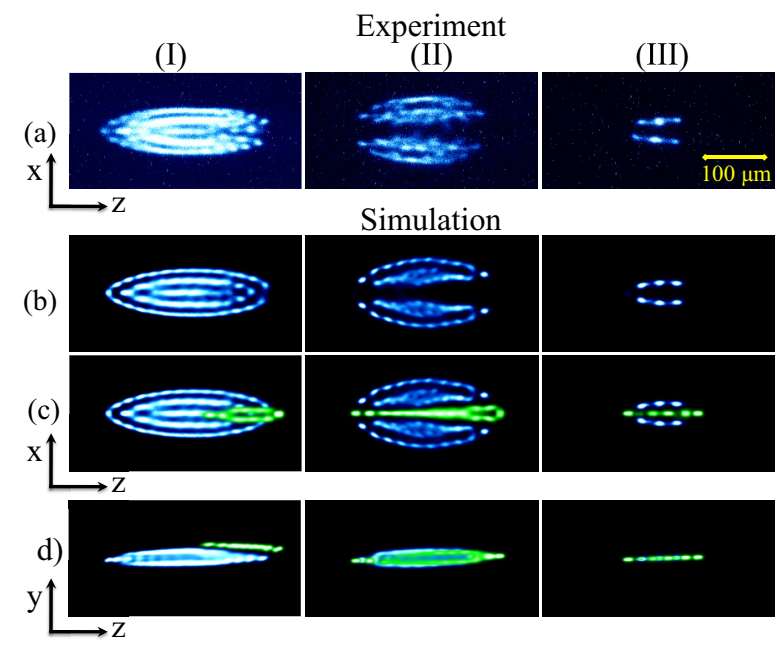

FIG. 4. (Color online) $\mathrm{Ca}^{+}-\mathrm{N}_{2}^{+}$bicomponent Coulomb crystals on the chip at different trapping configurations. (a) Experimental and (b-d) simulated false-color laser-cooling fluorescence images. $\mathrm{N}_{2}^{+}$ ions were made visible in green in the simulations for clarity. In (d), simulated images of the side view of the crystals in the $(y, z)$ plane perpendicular to the chip are shown. (I) $9 \mathrm{~N}_{2}^{+}$ions spatially separated from the $\mathrm{Ca}^{+}$crystal by $\approx 19 \mu \mathrm{m}$ in the vertical direction, (II) $18 \mathrm{~N}_{2}^{+}$ ions bisecting a near-planar Coulomb crystal with $50 \mathrm{Ca}^{+}$ions, and (III) a string of $5 \mathrm{~N}_{2}^{+}$ions sympathetically cooled by two strings with $3 \mathrm{Ca}^{+}$ions each.

In the experiment shown in column (II) of Fig. 4, a nearplanar bicomponent Coulomb crystal was formed with $18 \mathrm{~N}_{2}^{+}$ ions centered along the $\mathrm{rf}$ null line splitting the $\mathrm{Ca}^{+}$crystal in two halves. From the simulated side view of the crystal shown in Fig. 4(d), column (II), it can be seen that the crystal has a pancake-like shape and is at a maximum only two layers of ions thick.

Figure 4, column (III), shows a completely planar structure consisting of a string of five $\mathrm{N}_{2}^{+}$ions sympathetically cooled by two adjacent strings of three laser-cooled $\mathrm{Ca}^{+}$ ions each. Such planar bicomponent structures have recently been proposed as a framework for quantum computation and simulation [37].

In summary, we have reported the trapping and sympathetic cooling of molecular ions above the surface of an iontrap chip. The trapping potentials generated on the chip enabled the formation of two-layer and planar bicomponent Coulomb crystals. Capitalizing on the mass dependence of the pseudopotential, spatially separated layers of atomic and molecular ions could be formed. The flexibility and variability in designing electrode structures in SE traps $[6,38]$ pave the way for more sophisticated trapping architectures of benefit for molecular spectroscopy, chemistry, quantum technology, and mass spectrometry.

We gratefully acknowledge the technical support by Dieter Wild, Grischa Martin, Philipp Knöpfel, and Andreas Tonin. This work was supported by the University of Basel, the COST Action MP1001 "Ion Traps for Tomorrow's Applications," the Swiss National Science Foundation through the National Centre of Competence in Research "Quantum Science and Technology," and the Swiss Nanoscience Institute.
[1] Atom Chips, edited by J. Reichel and V. Vuletić (WILEY-VCH, Weinheim, Germany, 2011).

[2] J. Fortágh and C. Zimmermann, Science 307, 860 (2005).

[3] M. F. Riedel, P. Böhi, Y. Li, T. W. Hänsch, A. Sinatra, and P. Treutlein, Nature (London) 464, 1170 (2010).

[4] J. Chiaverini, R. B. Blakestad, J. Britton, J. D. Jost, C. Langer, D. Leibfried, R. Ozeri, and D. J. Wineland, Quantum Inf. Comput. 5, 419 (2005).

[5] D. Stick, W. K. Hensinger, S. Olmschenk, M. J. Madsen, K. Schwab, and C. Monroe, Nat. Phys. 2, 36 (2006).

[6] M. D. Hughes, B. Lekitsch, J. A. Broersma, and W. K. Hensinger, Contemp. Phys. 52, 505 (2011).

[7] D. Kielpinski, C. Monroe, and D. J. Wineland, Nature (London) 417, 709 (2002).

[8] C. Ospelkaus, U. Warring, Y. Colombe, K. R. Brown, J. M. Amini, D. Leibfried, and D. J. Wineland, Nature (London) 476, 181 (2011).

[9] R. J. Clark, Z. Lin, K. S. Diab, and I. L. Chuang, J. Appl. Phys. 109, 076103 (2011).

[10] B. Szymanski, R. Dubessy, B. Dubost, S. Guibal, J. P. Likforman, and L. Guidoni, Appl. Phys. Lett. 100, 171110 (2012).

[11] I. M. Buluta, M. Kitaoka, S. Georgescu, and S. Hasegawa, Phys. Rev. A 77, 062320 (2008).

[12] H. Loh, K. C. Cossel, M. C. Grau, K.-K. Ni, E. R. Meyer, J. L. Bohn, J. Ye, and E. A. Cornell, Science 342, 1220 (2013).
[13] J. J. Hudson, D. M. Kara, I. J. Sallman, B. E. Sauer, M. R. Tarbutt, and E. A. Hinds, Nature (London) 473, 493 (2011).

[14] S. Willitsch, M. T. Bell, A. D. Gingell, S. R. Procter, and T. P. Softley, Phys. Rev. Lett. 100, 043203 (2008).

[15] S. Ospelkaus, K.-K. Ni, D. Wang, M. H. G. de Miranda, B. Neyenhuis, G. Quéméner, P. S. Julienne, J. L. Bohn, D. S. Jin, and J. Ye, Science 327, 853 (2010).

[16] F. H. J. Hall and S. Willitsch, Phys. Rev. Lett. 109, 233202 (2012).

[17] M. Kirste, X. Wang, H. C. Schewe, G. Meijer, K. Liu, A. van der Avoird, L. M. C. Janssen, K. B. Gubbels, G. C. Groenenboom, and S. Y. T. van de Meerakker, Science 338, 1060 (2012).

[18] D. I. Schuster, L. S. Bishop, I. L. Chuang, D. DeMille, and R. J. Schoelkopf, Phys. Rev. A 83, 012311 (2011).

[19] D. DeMille, Phys. Rev. Lett. 88, 067901 (2002).

[20] J. Mur-Petit, J. J. García-Ripoll, J. Pérez-Ríos, J. CamposMartínez, M. I. Hernández, and S. Willitsch, Phys. Rev. A 85, 022308 (2012).

[21] E. S. Shuman, J. F. Barry, and D. DeMille, Nature (London) 467, 820 (2010).

[22] S. A. Meek, H. Conrad, and G. Meijer, Science 324, 1699 (2009).

[23] S. Marx, D. Adu Smith, M. J. Abel, T. Zehentbauer, G. Meijer, and G. Santambrogio, Phys. Rev. Lett. 111, 243007 (2013).

[24] S. D. Hogan, P. Allmendinger, H. Saßmannshausen, H. Schmutz, and F. Merkt, Phys. Rev. Lett. 108, 063008 (2012). 
[25] K. Mølhave and M. Drewsen, Phys. Rev. A 62, 011401 (2000).

[26] S. Willitsch, Int. Rev. Phys. Chem. 31, 175 (2012).

[27] D. Gerlich, in Advances in Chemical Physics, edited by C.-Y. Ng and M. Baer (Wiley, New York, 1992), Vol. 82, p. 1.

[28] D. T. C. Allcock, J. A. Sherman, D. N. Stacey, A. H. Burrell, M. J. Curtis, G. Imreh, N. M. Linke, D. J. Szwer, S. C. Webster, A. M. Steane, and D. M. Lucas, New J. Phys. 12, 053026 (2010).

[29] D. J. Berkeland, J. D. Miller, J. C. Bergquist, W. M. Itano, and D. J. Wineland, J. Appl. Phys. 83, 5025 (1998).

[30] J. M. Amini, H. Uys, J. H. Wesenberg, S. Seidelin, J. Britton, J. J. Bollinger, D. Leibfried, C. Ospelkaus, A. P. VanDevender, and D. J. Wineland, New J. Phys. 12, 033031 (2010).
[31] X. Tong, A. H. Winney, and S. Willitsch, Phys. Rev. Lett. 105, 143001 (2010).

[32] R. Schmied, New J. Phys. 12, 023038 (2010).

[33] R. Schmied, SurfacePattern software package, https://atom. physik.unibas.ch/people/romanschmied/code/SurfacePattern.php.

[34] C. B. Zhang, D. Offenberg, B. Roth, M. A. Wilson, and S. Schiller, Phys. Rev. A 76, 012719 (2007).

[35] M. T. Bell, A. D. Gingell, J. Oldham, T. P. Softley, and S. Willitsch, Faraday Discuss. 142, 73 (2009).

[36] A. Ostendorf, C. B. Zhang, M. A. Wilson, D. Offenberg, B. Roth, and S. Schiller, Phys. Rev. Lett. 97, 243005 (2006).

[37] I. M. Buluta and S. Hasegawa, Phys. Rev. A 78, 042340 (2008).

[38] R. Schmied, J. H. Wesenberg, and D. Leibfried, Phys. Rev. Lett. 102, 233002 (2009). 